\title{
RESPIRATORY INFECTIONS
}

\section{Asymptomatic carriage of Pneumocystis jiroveci in subjects undergoing bronchoscopy: a prospective study}

\author{
N A Maskell, D J Waine, A Lindley, J C T Pepperell, A E Wakefield, R F Miller, \\ R J O Davies
}

Thorax 2003;58:594-597

\begin{abstract}
Background: The opportunistic fungus Pneumocystis jiroveci is a common cause of respiratory infection in immunocompromised patients. By contrast, pneumocystis pneumonia (PCP) occurs only rarely in immunocompetent individuals. Asymptomatic colonisation with $P$ jiroveci has recently been described in patients who are either minimally immunosuppressed or who have underlying lung disorders such as bronchiectasis. We sought to determine the prevalence of asymptomatic colonisation by $P$ jiroveci in a cohort of adult patients undergoing diagnostic bronchoscopy.

Methods: A prospective observational cohort study was performed in patients who required bronchoscopy and bronchoalveolar lavage (BAL) as part of their routine clinical assessment. All the samples underwent standard microbiological analysis and a Grocott methenamine silver stain was performed where clinically indicated to detect the presence of $P$ jiroveci. Polymerase chain reaction for detection of $P$ jiroveci specific DNA was also performed. Results: Ninety three consecutive BAL fluid samples were analysed, 17 (18\%) of which contained $P$ jiroveci DNA. Of the potential predictors examined, only glucocorticoid use was significantly associated with detectable $P$ jiroveci DNA. Eighteen patients were receiving oral glucocorticoids (equivalent to $>20 \mathrm{mg}$ /day prednisolone) at the time of bronchoscopy, of whom eight $(44 \%)$ had detectable $P$ jiroveci DNA. In contrast, $P$ jiroveci was detected in only nine of 75 patients $(12 \%)$ who were not receiving glucocorticoids (difference between proportions 32\%, 95\% Cl 8 to $57 ; \mathrm{p}=0.004$, two tailed Fisher's exact test).

Conclusions: $P$ jiroveci colonisation, as determined by detection of $P$ jiroveci DNA in BAL fluid, is common in HIV negative patients with primary respiratory disorders undergoing bronchoscopy and BAL. The higher prevalence in patients receiving corticosteroids suggests that oral glucocorticoid therapy is an independent risk factor for colonisation. In contrast, underlying lung cancer or COPD did not appear to be risk factors.
\end{abstract}

See end of article for authors' affiliations

23 October 2002

Accepted for publication

3 April 2003

$\mathrm{T}$ he opportunistic fungus Pneumocystis jiroveci (formerly known as Pneumocystis carinii f. sp. hominis ${ }^{12}$ ) is a common cause of respiratory infection in immunocompromised patients, particularly those with HIV infection. ${ }^{3}$ By contrast, pneumocystis pneumonia (PCP) occurs only very rarely in immunocompetent individuals.

The route of transmission of human pneumocystis infection is unclear. ${ }^{2}$ Animal studies have demonstrated airborne transmission. ${ }^{24}$ P jiroveci has been identified in air spores ${ }^{25}$ and it is probable that transmission between persons occurs via the airborne route. ${ }^{26}$

New techniques such as the identification of $P$ jiroveci DNA by polymerase chain reaction (PCR) have recently been developed which allow the detection of very low levels of $P$ jiroveci not detectable by routine histochemical staining. ${ }^{27}$ These approaches have shown that $P$ jiroveci is carried in asymptomatic individuals with only mild immunosuppression induced by HIV infection, ${ }^{8}$ in patients requiring long term glucocorticoid therapy for underlying malignancy, and in immunocompetent individuals ${ }^{7}$ with chronic pulmonary diseases. ${ }^{9-11}$

In this prospective study we have determined the prevalence of asymptomatic colonisation by $P$ jiroveci in a cohort of adult patients undergoing diagnostic bronchoscopy and lavage. We further sought to identify risk factors associated with colonisation.

\section{METHODS}

\section{Study design and setting}

This prospective observational cohort study was performed at the Oxford Centre for Respiratory Medicine. Patients are referred to the centre directly by general practitioners $(60 \%)$, by other local hospital consultants (35\%), and occasionally by respiratory consultants in other neighbouring health authorities $(<5 \%)$. The hospital has a large regional renal transplant unit and oncology centre and covers a population of 500000. Consecutive patients attending the chest unit who required diagnostic bronchoscopy as part of their standard management for possible respiratory disease were recruited over a 24 month period. All patients undergoing bronchoscopy at this time had a bronchoalveolar lavage (BAL) with 100-150 ml normal saline as part of the routine clinical practice. The study was performed within the guidelines of the Central Oxford Research Ethics Committee.

\section{Subjects and follow up}

Patients were eligible if they required bronchoscopy as part of their routine clinical assessment. None of the subjects was known to be HIV positive, but HIV testing was not performed routinely as part of the study protocol. Ineligibility criteria were: patients known to be HIV positive, age $<18$ years, and inability to give informed consent. Patients were followed for 2 years or until death (in cases of lung malignancy). All the samples underwent standard microbiological analysis and, where clinically indicated, a Grocott methenamine silver stain was performed on BAL fluid samples to detect the presence of P jiroveci.

\section{Procedure}

At the time of diagnostic bronchoscopy a $6 \mathrm{ml}$ aliquot of BAL fluid was frozen at $-80^{\circ} \mathrm{C}$ and subsequently transferred to the 
Table 1 Final respiratory diagnosis in the 93 patients

\begin{tabular}{ll}
\hline & No (\%) \\
\hline Lung cancer & $35(38 \%)$ \\
Pneumonia & $24(26 \%)$ \\
Vasculitis & $2(2 \%)$ \\
Prior inactive tuberculosis & $6(6 \%)$ \\
Aspergillus lung disease & $2(2 \%)$ \\
No respiratory diagnosis & $6(6 \%)$ \\
Other & $18(19 \%)$ \\
Total & 93 \\
\hline
\end{tabular}

Weatherall Institute of Molecular Medicine for analysis. The BAL fluid samples were coded and analyses were carried out blind to the patients' details.

\section{Detection of $P$ iiroveci DNA in BAL fluid samples}

BAL fluid samples were pooled in batches of four samples. DNA was extracted from the pooled samples with a QIAmp mini kit (Qiagen, Hilden, Germany) using the manufacturer's protocol with the following modifications: (1) proteinase K digestion was performed for 1 hour rather than 10 minutes, and (2) DNA was eluted from the column in $50 \mu \mathrm{l}$ rather than $200 \mu \mathrm{l}$ AE buffer.

DNA amplification at the mitochondrial large subunit rRNA (mt LSU rRNA) was performed using a nested PCR with the primers pAZ102-H and pAZ102-E in the first round amplification and the primers pAZ102-X $\left(5^{\prime}\right.$ GTGAAATACAAATCGGACTAGG- $\left.3^{\prime}\right)$ and pAZ102-Z (5'AAGCCCACTTCTTRACCTGTC-3') in the second round (where $\mathrm{R}=\mathrm{T}$ or $\mathrm{C}$ ), as previously described..$^{72-14}$ If a positive PCR reaction was obtained from any batch, a further extraction of DNA was carried out on each individual BAL fluid sample in that batch, as described above. ${ }^{72-14}$ Extreme care was taken to eliminate the possibility of cross contamination of samples, including the inclusion of negative controls in each extraction, the handling of samples in a laminar flow cabinet, and the use of disposable tips, tubes and aliquots of reagents. Nested PCR at the mt LSU rRNA was then performed on each of the individually extracted samples, as described above. In each amplification experiment a sample of $P$ jiroveci DNA obtained from a patient with histologically confirmed PCP was used as a positive control. Taq DNA polymerase (Promega, Southampton, UK) was used throughout the study.

In BAL fluid samples with no detectable $P$ jiroveci DNA a control PCR was performed, using the conserved human $\beta$-globin gene sequence, to monitor for false negative PCR reactions caused by inhibition of DNA amplification. A positive result using PCR for human $\beta$-globin with the primers BGLO-1 (5'-ACACAATGTGTTCACTAGCA-3') and BGLO-2 (5'CAACTTCATCCACGTTCAC-C- $\left.3^{\prime}\right)^{15}$ demonstrated that DNA amplification was possible on a given sample and that the negative PCR for $P$ jiroveci was not due to inhibition of the reaction.

\section{Sequencing of $P$ jiroveci DNA}

The products of PCR amplification at the mt LSU rRNA were purified using the SpinPrep PCR Clean-up kit (Novagen, Nottingham, UK) and sequenced directly using the Big Dye Terminator Cycling Sequencing Ready Reaction Kit, version 2.0 (Applied Biosystems, Warrington, UK) and the ABI 377 DNA sequencer running the data collection software version 2.1 (Applied Biosystems). Sequence data analyses were performed with Chromas 1.62 software (Technelysium Pty, Australia) and the University of Wisconsin Genetics Computer Group software, version 10.1 (Genetics Computer Group, Madison, Wisconsin, USA). Polymorphisms at nucleotide positions 85 and 248 were used to describe genotypes of $P$ jiroveci, as described previously. ${ }^{12}$

\section{Data analysis}

The primary outcome was the prevalence of $P$ jiroveci detected by PCR in BAL fluid. All analyses were performed with SPSS version 10 (SPSS Inc, Chicago, USA). The characteristics of patients with detectable $P$ jiroveci and patients with no detectable $P$ jiroveci were compared using $\chi^{2}$ analysis and Fisher's exact test was used where appropriate. A p value of $<0.05$ was considered significant.

\section{RESULTS}

Ninety three subjects of median age 65 years (range 20-87) participated in the study. There were 60 men (65\%) and 23 (25\%) had chronic obstructive pulmonary disease (COPD; mean (SD) forced expiratory volume in 1 second $\left(\mathrm{FEV}_{1}\right) 2.0$ (1.0) l, forced vital capacity (FVC) 2.8 (1.3) l); 23/86 (27\%) were current smokers and $42(49 \%)$ were ex-smokers. The final respiratory diagnoses of these 93 subjects are shown in table 1.

Ninety three BAL fluid samples were analysed for the presence of $P$ jiroveci by PCR and also by routine histochemical staining; 17 samples ( $18 \%$, CI 12 to 30 ) were positive by PCR and only one was also positive with histochemical staining. This patient received treatment with co-trimoxazole but died 8

\begin{tabular}{|c|c|c|c|c|c|}
\hline Patient no & Age & Sex & $\begin{array}{l}\text { Peripheral blood } \\
\text { lymphocyte count }\end{array}$ & $\begin{array}{l}\text { Oral } \\
\text { steroids }\end{array}$ & Final diagnoses \\
\hline 1 & 76 & M & 1.91 & & Prior inactive TB \\
\hline 2 & 20 & M & 1.74 & & smoke inhalation \\
\hline $3^{*}$ & 54 & M & 2.10 & & Lung cancer \\
\hline 4 & 78 & M & 2.23 & & Pneumonia, prostate cancer \\
\hline 5 & 51 & M & 1.10 & & Pneumonia \\
\hline 6 & 74 & $\mathrm{~F}$ & 0.65 & & Lung cancer \\
\hline 7 & 74 & $\mathrm{~F}$ & 0.55 & & Lung cancer \\
\hline 8 & 69 & M & 2.40 & Yes & Lung cancer \\
\hline 9 & 51 & M & 1.10 & Yes & Lung cancer \\
\hline 10 & 58 & M & 10 & Yes & Aspergilloma + CLL \\
\hline 11 & 75 & M & 1.10 & Yes & MAl \\
\hline 12 & 70 & M & & Yes & Lung cancer + Wegener's \\
\hline 13 & 65 & $\mathrm{~F}$ & & Yes & Prior inactive TB \\
\hline 14 & 63 & M & 1.87 & Yes & Pneumonia \\
\hline 15 & 69 & M & 1.12 & Yes & Prior inactive $\mathrm{TB}+$ renal transplan \\
\hline 16 & 28 & M & 2.40 & & Pneumonia \\
\hline 17 & 40 & M & 3.77 & & Pneumonia \\
\hline
\end{tabular}


Table 3 Comparison of subjects with and without detectable $P$ jiroveci DNA in BAL fluid

\begin{tabular}{llll}
\hline & $\begin{array}{l}\text { P jiroveci DNA not } \\
\text { detected }(n=76)\end{array}$ & $\begin{array}{l}\text { P jiroveci DNA } \\
\text { detected }(n=17)\end{array}$ & p value \\
\hline Use of oral glucocorticoids (prednisolone & $10(13 \%)$ & $8(47 \%)$ & 0.006 \\
$>20 \mathrm{mg} /$ day) & $65(28-87)$ & $65(20-78)$ & NS \\
Median (range) age (years) & $46(61 \%)$ & $14(82 \%)$ & $\mathrm{NS}$ \\
Sex (\% male) & $19(25 \%)$ & $4(24 \%)$ & $\mathrm{NS}$ \\
COPD & $6(8 \%)$ & $2(12 \%)$ & $\mathrm{NS}$ \\
On immunosuppressant drugs (excluding & $7(41 \%)$ & $\mathrm{NS}$ \\
glucocorticoids) & $29(38 \%)$ & & \\
Cancer & & & \\
\hline NS=not significant (p>0.05). & & &
\end{tabular}

weeks later from metastatic lung carcinoma. Table 2 shows the characteristics of the 17 patients who were positive for $P$ jiroveci by PCR.

Eighteen patients (19\%) were receiving oral glucocorticoids equivalent to $>20 \mathrm{mg} /$ day prednisolone at the time of bronchoscopy, eight of whom (44\%) had detectable $P$ jiroveci. In contrast, $P$ jiroveci was detected in only nine of 75 patients $(13 \%)$ who were not receiving glucocorticoids (difference between proportions $32 \%, 95 \%$ CI 8 to $57, p=0.004$, Fisher's exact test).

There were no significant differences between those with and without detectable $P$ jiroveci in age, sex, or lung function ( $\mathrm{FEV}_{1}$ and FVC). However, immunosuppression (due to glucocorticoids, cyclophosphamide, azathioprine, or haematological malignancy) was significantly more likely in the subjects with detectable $P$ jiroveci in the BAL fluid $(\mathrm{p}=0.02$, Fisher's exact test, table 3 ).

\section{DISCUSSION}

In this prospective study of adult patients, $P$ jiroveci was detected by PCR in the BAL fluid of 17 of 93 patients (18\%). In the subgroup of patients who were not receiving oral glucocorticoids or other immunosuppressants $(\mathrm{n}=73), P$ jiroveci was detected in nine (12\%) compared with eight (40\%) of the 20 patients receiving immunosuppressive agents. The presence of COPD or lung cancer was not associated with an increased likelihood of detecting $P$ jiroveci in the BAL fluid.

The high frequency of detection of $P$ jiroveci in BAL fluid from unselected patients undergoing routine bronchoscopy in this study suggests that the incidence of colonisation with $P$ jiroveci in hospital patients-and perhaps the general population-may be higher than previously thought. The significance of this colonisation is unknown at present, although it may represent a biological reservoir of infection which can be transmitted to more susceptible hosts.

All 24 patients with a final diagnosis of pneumonia had new onset of fever with focal or diffuse chest radiographic abnormalities, with or without purulent sputum production. All responded to specific antibiotics in conventional doses. In only a small number of these patients was a pathogen identified from the sputum, BAL fluid and/or the blood.

None of the 17 patients with detectable $P$ jiroveci developed PCP during the 2 year follow up period. This is reassuring since many of these patients were found to have primary lung malignancy and often received chemotherapy shortly after their diagnostic bronchoscopy. The underlying frequency of COPD and lung cancer was similar in those with and without detectable $P$ jiroveci, which suggests that these are not independent risk factors for $P$ jiroveci colonisation, a finding consistent with previously published studies. ${ }^{16}{ }^{17}$ The level of colonisation in HIV negative immunocompetent patients ranges from $6 \%$ to $19 \%,{ }^{16}{ }^{17}$ but these reports do not clearly describe the nature of the underlying conditions nor do they give details of the dose of corticosteroid used. In this study patients receiving oral corticosteroids (>20 mg/day prednisolone) were significantly more likely to have detectable $P$ jiroveci $(44 \%)$ than patients who were not receiving glucocorticoids ( $12 \% ; p=0.004$, Fisher's exact test). This finding is supported by those of previous studies. ${ }^{318} 19$ All of this subgroup were receiving $>20 \mathrm{mg} /$ day prednisolone at the time of the bronchoscopy. Most of the patients were on short courses of glucocorticoid therapy but several were on long term therapy for a variety of disorders.

Glucocorticoids induce marked changes in pulmonary surfactant composition, with reductions in phospholipids and increases in both phospholipase A2 and surfactant proteins A and D. ${ }^{20}{ }^{21}$ It has been shown both in vitro and in non-steroid immunosuppressed animal models of Pneumocystis infection that proliferation of Pneumocystis in the lung requires changes in surfactant (phospholipid and protein) composition. ${ }^{22}$ It is therefore possible that glucocorticoid induced changes in surfactant composition in our patients may have facilitated colonisation with $P$ jiroveci.

A previous report has shown that pulmonary colonisation with $P$ jiroveci occurred mainly in HIV negative patients with underlying disease and defects of CD4 T cell function..$^{23}$ In this study CD4 lymphocyte counts were not routinely measured. It is noteworthy that the total lymphocyte counts in some patients were below the normal range, but there were no differences between those with and without detectable $P$ jiroveci.

This study leaves some questions unanswered. Further studies are needed to determine whether patients who are colonised by $P$ jiroveci are infectious to immunocompromised patients, and whether colonisation by $P$ jiroveci leads to PCP directly or whether the pneumonia is caused by a new airborne infection.

\section{Authors' affiliations}

N A Maskell, D J Waine, J C T Pepperell, R J O Davies, Oxford Centre for Respiratory Medicine, Churchill Hospital, Oxford Radcliffe NHS Trust, Oxford OX3 7L, UK

A Lindley, A E Wakefield, Department of Paediatrics, Weatherall Institute of Molecular Medicine, Oxford Raddliffe NHS Trust, Oxford OX3 9DU, UK

R F Miller, Windeyer Institute of Medical Sciences, Royal Free and University College Medical School, University College London, London, UK

Conflict of interest: none.

Funding: none.

\section{REFERENCES}

1 Stringer JR, Cushion MT, Wakefield AE. New nomenclature for the genus Pneumocystis. J Euk Microbiol 2002;49:184-9S.

2 Stringer JR, Beard CB, Miller RF, et al. A new name (Pneumocystis jiroveci) for Pneumocystis from humans. Emerg Infect Dis 2002;8:891-6.

3 Kovacs JA, Gill VJ, Meshnick S, et al. New insights into transmission, diagnosis, and drug treatment of Pneumocystis carinii pneumonia. JAMA 2001;286:2450-60.

4 Dumoulin A, Mazars E, Seguy N, et al. Transmission of Pneumocystis carinii disease from immunocompetent contacts of infected hosts to susceptible hosts. Eur J Clin Microbiol Infect Dis 2000; 19:671-8. 
5 Wakefield AE. DNA sequences identical to Pneumocystis carinii f. sp. carinii and Pneumocystis carinii f. sp. hominis in samples of air spora. J Clin Microbiol 1996;34:1754-9.

6 Miller RF, Ambrose HE, Novelli V, et al. Probable mother-to-infant transmission of Pneumocystis carinii f. sp. hominis infection. J Clin Microbiol 2002:40: 1555-7.

7 Miller RF, Ambrose HE, Wakefield AE. Pneumocystis carinii f. sp. hominis DNA in immunocompetent health care workers in contact with patients with P. carinii pneumonia. J Clin Microbiol 2001;39:3877-82.

8 Nevez G, Raccurt C, Jounieaux V, et al. Pneumocystosis versus pulmonary Pneumocystis carinii colonization in HIV-negative and HIV-positive patients. AIDS 1999;13:535-6.

9 Henson JW, Jalaj JK, Walker RW, et al. Pneumocystis carinii pneumonia in patients with primary brain tumors. Arch Neurol 1991;48:406-9.

10 Calderon EJ, Regordan C, Medrano FJ, et al. Pneumocystis carinii infection in patients with chronic bronchial disease. Lancet 1996;347:977.

11 Peters SG, Prakash UB. Pneumocystis carinii pneumonia. Review of 53 cases. Am J Med 1987;82:73-8.

12 Tsolaki AG, Beckers P, Wakefield AE. Pre-AIDS era isolates of Pneumocystis carinii f. sp. hominis: high genotype similarity with contemporary isolates. J Clin Microbiol 1998;36:90-3.

13 Wakefield AE, Pixley FJ, Banerii S, et al. Detection of Pneumocystis carinii with DNA amplification. Lancet 1990;336:451-3.

14 Palmer RJ, Settnes OP, Lodal J, et al. Population structure of rat-derived Pneumocystis carinii in Danish wild rats. Appl Environ Microbiol 2000;66:4954-61
15 Saiki RK, Scharf S, Faloona F, et al. Enzymatic amplification of beta-globin genomic sequences and restriction site analysis for diagnosis of sickle cell anemia. Science 1985;230:1350-4.

16 Sing A, Roggenkamp A, Autenrieth IB, et al. Pneumocystis carinii carriage in immunocompetent patients with primary pulmonary disorders as detected by single or nested PCR. J Clin Microbiol 1999;37:3409_ as dete.

17 Armbruster C, Hassl A, Kriwanek S. Pneumocystis carinii colonization in the absence of immunosuppression. Scand J Infect Dis 1997;29:591-3.

18 Miller RF. Pneumocystis carinii in non-AIDS patients. Curr Opin Infect Dis 1999;12:371-7.

19 Jacobs JL, Libby DM, Winters RA, et al. A cluster of Pneumocystis carinii pneumonia in adults without predisposing illnesses. N Engl J Med 1991:324:246-50.

20 Deterding RR, Schimizu H, Fisher $\mathrm{JH}$, et al. Regulation of surfactant protein $\mathrm{D}$ expression by glucocorticoids in vitro and in vivo. Am J Respir Cell Mol Biol 1994;10:30-7.

21 Guo Z, Kaneshiro ES. Phospholipid composition of Pneumocystis carinii carinii and effects of methylprednisolone immunosuppression on rat lung lipids. Infect Immun 1995:63:1286-90.

22 Aliouat EM, Escamilla R, Cariven C, et al. Surfactant changes during experimental pneumocystosis are related to pneumocystis development Eur Respir J 1998;1 1:542-7.

23 Nevez G, Raccurt $C$, Vincent $P$, et al. Pulmonary colonization with Pneumocystis carinii in human immunodeficiency virus-negative patients: assessing risk with blood CD4+ T cell counts. Clin Infect Dis 1999;29:1331-2.

\section{LUNG ALERT}

\section{Acetylcholine may be an autocrine growth factor for lung cancer}

A Song P, Sekhon HS, Proskocil B, et al. Synthesis of acetylcholine by lung cancer. Life Sci 2003;72:2159-68

$\mathrm{R}$ esearchers have recently shown that small cell lung cancer (SCLC) cell lines secrete acetylcholine (ACh) into tissue culture medium. Treatment of the cell lines in vitro with either muscarinic (atropine) or nicotinic (mecalmylamine) antagonists reduced cell growth in cell lines that produced high quantities of ACh. This effect of ACh inhibition was seen with both inhibitors but not with cell lines that had a low baseline level of ACh secretion. Previous data from the same group (Cancer Res 2003;63:214-2) found that 13 of 26 archival SCLC expressed the biosynthetic enzyme for ACh production, choline acetyltransferase. These studies show that SCLC can synthesise and secrete ACh, and that released non-neuronal ACh stimulates SCLC cell growth. Identification of this autocrine loop provides a potential new avenue for therapeutic intervention in SCLC cell lines that are high ACh secretors. These data also reinforce the importance of smoking cessation, given the potential of exogenous nicotine to promote tumour growth.

A De-Soyzo 